Pacific Journal of Mathematics

SEMIPERFECT RINGS WITH ABELIAN ADJOINT GROUP 


\title{
SEMIPERFECT RINGS WITH ABELIAN ADJOINT GROUP
}

\section{W. KEITH NICHOLSON}

\begin{abstract}
A structure theorem is proved for semiperfect rings (possibly with no identity) which have an abelian adjoint group. This is used to give conditions when such a ring is finite or commutative. In particular, a semiperfect ring with identity is finite if its group of units is finitely generated and abelian. Additional information is obtained if the adjoint group is cyclic.
\end{abstract}

1. Preliminaries. Throughout this paper all rings will be associative but need not contain an identity element. If $R$ is a ring, the adjoint group of $R$ is the set $R^{0}$ of all elements of $R$ which have inverses with respect to the operation $a \circ b=a+b-a b$. This operation will be called adjoint multiplication. If $R$ has an identity the multiplicative group of units of $R$ will be denoted by $R^{*}$. It is well known that $R^{*}$ and $R^{0}$ are isomorphic groups. The additive group of a ring $R$ will be denoted by $R^{+}$.

If $R$ is a ring, a left $R$-module $X$ will be called $G$-unital if $R^{0} X=0$. If $R$ has an identity this is equivalent to the condition that $u x=x$ for all $u \in R^{*}$ and $x \in X$, and so agrees with the usage of this term in [4]. A bimodule is $G$-unital if it is $G$-unital on both sides.

Let $S$ and $A$ be rings, let $X$ be an $S-A$ bimodule and let $Y$ be an $A-S$ bimodule. The semidirect sum $\left[\begin{array}{cc}S & X \\ Y & A\end{array}\right]$ is defined to be the set of all $2 \times 2$ "matrices" with components as shown. This is a ring if we use componentwise addition and multiplication

$$
\left(\begin{array}{ll}
s & x \\
y & a
\end{array}\right)\left(\begin{array}{ll}
s^{\prime} & x^{\prime} \\
y^{\prime} & a^{\prime}
\end{array}\right)=\left(\begin{array}{cc}
s s^{\prime} & s x^{\prime}+x a^{\prime} \\
y s^{\prime}+a y^{\prime} & a a^{\prime}
\end{array}\right) .
$$

The next proposition characterizes the adjoint group of such a ring and its routine proof is left to the reader.

Proposition 1. Let $S$ and $A$ be rings, let $X$ be an $S-A$ bimodule and let $Y$ be an $A-S$ bimodule. Then $\left[\begin{array}{cc}S & X \\ Y & A\end{array}\right]^{0}=\left[\begin{array}{cc}S^{0} & X \\ Y & A^{0}\end{array}\right]$ and this group is abelian if and only if $S^{0}$ and $A^{0}$ are abelian and both $X$ and $Y$ are G-unital. Moreover, when this is the case the adjoint group $\left[\begin{array}{cc}S & X \\ Y & A\end{array}\right]^{0}$ is isomorphic to the direct product of the adjoint groups $S^{0}$ and $A^{0}$ and the additive groups $X$ and $Y$.

2. The structure theorem. The Jacobson radical of a ring $R$ 
will be denoted by $J(R)$. A ring $R$ (possibly with no identity) will be called semiperfect if $R / J(R)$ is semisimple and idempotents can be lifted modulo $J(R)$. A ring $R$ is called local if it has an identity and has a unique maximal left ideal. An idempotent $e^{2}=e \in R$ is called a local idempotent if the ring $e R e$ is local.

Suppose now that $R$ is a semiperfect ring with $R^{0}$ abelian. Then we may choose an idempotent $e \in R$ such that $e=e_{1}+e_{2}+\cdots+e_{n}$ where the $e_{i}$ are orthogonal local idempotents and $\bar{e}=e+J(R)$ is the identity of $R / J(R)$. If $R$ has an identity then, necessarily, $e=1$. We shall use the following notation:

$$
\begin{aligned}
S & =e R e \\
X & =\{x \in R \mid e x=x, x e=0\} \\
Y & =\{y \in R \mid y e=y, e y=0\} \\
A & =\{a \in R \mid e a=0=a e\} .
\end{aligned}
$$

Clearly $S$ and $A$ are rings ( $S$ with identity), $X$ is an $S-A$ bimodule and $Y$ is an $A-S$ bimodule.

Lemma 1. Suppose $R$ is a semiperfect ring with $R^{0}$ abelian. Then:

(1) $S$ is a semiperfect ring with identity and $S^{*}$ is abelian.

(2) $A$ is a commutative ring with $J(A)=A$.

Proof. The identity $e$ of $S$ is the sum of the orthogonal local idempotents $e_{i} \in S$ so $S$ is semiperfect by a result of Mueller ([3], Theorem 1). We have $S^{*} \cong S^{0} \cong R^{0}$ so $S^{*}$ is abelian, proving (1). If $\mathrm{a} \in A$ then $\bar{a}=\bar{a} \bar{e}=\bar{e} \bar{a}=\overline{0}$ in $R / J(R)$ and it follows that $A \subseteq J(R)$. This implies $A$ is commutative since $R^{0}$ is abelian. If $a \in A$, let $b \in R$ satisfy $a \circ b=0$. Then $b=a b-a$ so $e b=0$. Similarly $b e=0$ so $b \in A$. This implies $J(A)=A$.

Lemma 2. Let $R$ be a ring with $R^{0}$ abelian. If $e^{2}=e \in R$ the identities erse $=$ erese and ser $=$ eser + sere - esere hold for all elements $r$ and $s$ in $R$.

Proof. Write $a=e r$ - ere and $b=s e-e s e$. Then $a^{2}=0=b^{2}$ so that $a, b \in R^{0}$. It follows that $a b=b a$ and hence that $a b=(e a) b=$ $e b a=0$. But $a b=$ erse - erese and $b a=$ ser - sere - eser + esere.

Now define a map from $R$ to $\left[\begin{array}{cc}S & X \\ Y & A\end{array}\right]$ by

$$
r \longmapsto\left(\begin{array}{cc}
e r e & e r-e r e \\
r e-e r e & r-e r-r e+e r e
\end{array}\right) \text {. }
$$


This is obviously a monomorphism of additive abelian groups and it is onto since an element $\left(\begin{array}{ll}s & x \\ y & a\end{array}\right) \in\left[\begin{array}{ll}S & X \\ Y & A\end{array}\right]$ is the image of $r=s+x+y+a$. Lemma 2 guarantees that it is a ring isomorphism as an easy calculation shows. Hence $R$ is represented as a semidirect sum and so Proposition 1 and the fact that $R^{0}$ is abelian show that $X$ and $Y$ are $G$-unital bimodules. But $A^{0}=A$ by Lemma 1 so $X A=0=A Y$. This in turn shows that $\left[\begin{array}{cc}S & X \\ Y & A\end{array}\right] \cong\left[\begin{array}{cc}S & X \\ Y & 0\end{array}\right] \oplus A$ using the definition of multiplication in the semidirect sum.

Now we consider the structure of the $G$-unital $S$-modules $X$ and $Y$. The identity of $S$ can be written $1=e_{1}+\cdots+e_{n}$ where the $e_{i}$ are orthogonal local idempotents. Moreover, the local rings $e_{i} S e_{i}$ are commutative since they have abelian adjoint groups. Since the $e_{i}$ are orthogonal we have a direct sum $X=e_{1} X \oplus \cdots \oplus e_{n} X$ of abelian groups. Moreover, $e_{i} X$ is a $G$-unital $e_{i} S e_{i}$-module for each $i$. Suppose $e_{i} \neq e_{j}$ and $s \in S$. Then $\left(e_{i} s e_{j}\right)^{2}=0$ so $e_{i} s e_{j} X=0$ because $X$ is $G$-unital. Hence, for each $s \in S, s x=\left(e_{1} s e_{1}\right) e_{1} x+\cdots+\left(e_{n} s e_{n}\right) e_{n} x$ holds for every $x \in X$. On the other hand, if $X_{i}$ is a $G$-unital $e_{i} S e_{i}$-module for each $i$ and if $X=X_{1} \oplus \cdots \oplus X_{n}$, then Lemma 2 guarantees that $X$ becomes a $G$-unital $S$-module if we define

$$
s\left(x_{1}, x_{2}, \cdots, x_{n}\right)=\left(e_{1} s e_{1} x_{1}, e_{2} s e_{2} x_{2}, \cdots, e_{n} s e_{n} x_{n}\right)
$$

for each $s \in S$ and $\left(x_{1}, \cdots, x_{n}\right) \in X$. This shows that the structure of the $G$-unital $S$-modules is completely determined up to the structure of the $G$-unital $e_{i} S e_{i}$-modules.

Suppose $e_{1}$ is such that the local ring $e_{1} S e_{1}$ has no nonzero $G$ unital module. It was proved in [4] that $e_{1}$ is central in $S$ so we can write $S=e_{1} S e_{1} \oplus S_{1}$. Furthermore, $e_{1} X=0$ and $Y e_{1}=0$ since these are $G$-unital $e_{1} S e_{1}$-modules. It follows from the preceding paragraph that $\left[\begin{array}{cc}S & X \\ Y & 0\end{array}\right] \cong e_{1} S e_{1} \oplus\left[\begin{array}{cc}S_{1} & X \\ Y & 0\end{array}\right]$. In this way each local ring $e_{2} S e_{i}$ which has no nonzero $G$-unital module splits off as a direct summand of $R$. Thus we may assume that $S$ is indecomposable.

On the other hand, a local ring $L$ which has a nonzero $G$-unital module must satisfy $L / J(L) \cong Z_{2}$, the ring of integers modulo 2 . Moreover, the $G$-unital $L$-modules are precisely the elementary abelian 2 -groups $X$ and the action is given by $a x=0$ or $x$ according as $a \in J(L)$ or $a \in L^{*}$. These will be referred to as trivial $L$-modules. Finally, if $L^{0}$ is abelian the ring $L$ must be commutative. The easy verification of these facts can be found in Proposition 2 of [4].

We summarize the results of this discussion in the following theorem.

THEOREM 1. If $R$ is a semiperfect ring (possibly with no identity) 
with abelian adjoint group $R^{0}$ then $R \cong T \oplus A \oplus\left[\begin{array}{cc}S & X \\ Y & 0\end{array}\right]$ where $T$ is a finite direct sum of commutative local rings, $A$ is a commutative ring with $J(A)=A$ and $S$ is an indecomposable semiperfect ring with identity such that $S^{*}$ is abelian and $X$ and $Y$ are G-unital $S$-modules. Conversely every such ring is semiperfect with abelian adjoint group. Furthermore:

(1) The identity of $S$ can be written $1=e_{1}+\cdots+e_{n}$ where the $e_{i}$ are orthogonal local idempotents and $e_{i} S e_{i} / J\left(e_{i} S e_{i}\right) \cong Z_{2}$ for each $i$. The module $X$ has the form $X=X_{1} \oplus \cdots \oplus X_{n}$ where $X_{i}$ is a trivial $e_{i} S e_{i}$-module for each $i$ and $s\left(x_{1}, \cdots, x_{n}\right)=\left(e_{1} s e_{1} x_{1}, \cdots, e_{n} s e_{n} x_{n}\right)$ for each $s \in S$ and $\left(x_{1}, \cdots, x_{n}\right) \in X$. The module $Y$ has an analogous structure.

(2) The adjoint group of $R$ is isomorphic to the direct product of the multiplicative groups $T^{0}, A^{0}$, and $S^{0}$ and the additive groups $X$ and $Y$.

The ring $S$ is characterized in [4] (Theorem 1) up to the structure of the commutative local rings $e_{i} S e_{i}$. Hence the present theorem completely characterizes all semiperfect rings $R$ with abelian adjoint group up to the structure of commutative local rings and commutative rings $A$ with $J(A)=A$. Moreover, the adjoint groups of these rings are direct factors of the adjoint groups of $R$ and so they inherit many other properties which $R^{0}$ may possess. In the cyclic case this leads to a complete characterization and this will be given in $\S 2$. But first we look at some other consequences of Theorem 1 .

CoROLLARY'1. Let $R$ be a semiperfect ring with abelian adjoint group. Then $R$ is commutative if either of the following conditions is satisfied:

(1) $2 x=0$ in $R$ implies $x=0$.

(2) $R^{0}$ has no direct factor each element of which has order 3.

Proof. Using the notation of Theorem 1, these conditions apply to $S$ so it is commutative by Corollary 1 of [4]. Both conditions imply $X=0=Y$ since these are (additive) elementary 2-groups. The result follows.

Our next result applies a result of Watters [6] which states that if $A$ is a ring with $J(A)=A$ then $A^{0}$ is a finitely generated nilpotent group if and only if $A^{+}$is a finitely generated group. Moreover, when these conditions are satisfied $A$ is a nilpotent ring ([6], Corollary 2).

Lemma 3. A local ring $R$ has a finitely generated abelian ad- 
joint group if and only if $R$ is finite and commutative.

Proof. The division ring $R / J(R)$ has a finitely generated abelian group of units and hence is finite. Since $R^{\circ}$ is abelian it follows that $R^{*}$ is an abelian group and $J(R)$ is a commutative ring. Since $R$ is local, this means it is a commutative ring. Moreover, $R$ is noetherian since any ascending chain of ideals is an ascending chain of adjoint subgroups. It follows that $J(R)^{n} / J(R)^{n+1}$ is a finite ring for each $n$ since it is a finite dimensional vector space over $R / J(R)$. But Watters' theorem asserts that $J(R)$ is nilpotent since $J(R)^{0}$ is a finitely generated abelian group. Hence $R$ is finite.

We remark here that this result in the cyclic case is proved in [4] without appealing to Watters' theorem.

Corollary 2. Let $R$ be a semiperfect ring. If the adjoint group $R^{0}$ is finitely generated and abelian, then $R \cong F \oplus A$ where $F$ is a finite ring and $A$ is a commutative nilpotent ring such that $A^{+}$ is finitely generated. In particular, a semiperfect ring with identity is finite if its group of units is finitely generated and abelian.

Proof. The last statement follows from Lemma 3 and Theorem 1 of [4]. Hence, using the notation of the theorem, $S$ is finite since $S^{0}$ is finitely generated. Lemma 3 implies that $T$ is finite and $X$ and $Y$ are finitely generated vector spaces over $Z_{2}$ and so are finite. The results of Watters show that $A$ is nilpotent and $A^{+}$is finitely generated.

Theorem 1 also gives the following result of Fischer and Eldridge ([1], Theorem 1).

COROLlary 3. If an artinian ring $R$ has a finitely generated abelian adjoint group then $R$ is finite.

Proof. Since $R$ is semiperfect we need only show that the ring $A$ in Corollary 2 is finite. An induction on the order of nilpotence of the ring $A$ shows that $A^{+}$satisfies the descending chain condition on subgroups (Szele [5], Theorem 1). But then $A^{+}$has a composition series and so is finite.

3. Cyclic adjoint groups. In this section we give a complete characterization of semiperfect rings with a cyclic adjoint group. This extends the results of Fischer and Eldridge ([1], Theorem 5). The first result we need is

Lemma 4. Let $A$ be a ring such that $J(A)=A$ and $A^{0}$ is cyclic. 
Either $A$ is finite or $A^{+}$is cyclie and $A^{2}=0$.

Proof. The ring $A$ is commutative and is nilpotent by Watters' theorem [6]. Suppose then that $A^{n} \neq 0$ and $A^{n+1}=0$. Assume $A^{0}$ is infinite cyclic. We must show that $n=1$. We have $\left(A^{i}\right)^{+} /\left(A^{i+1}\right)^{+} \cong$ $\left(A^{i}\right)^{0} /\left(A^{i+1}\right)^{0}$ so that, in particular, $\left(A^{n}\right)^{+} \cong\left(A^{n}\right)^{0}$ is infinite cyclic.

Assume $n>1$ and let $0 \neq a_{1} a_{2} \cdots a_{n} \in A^{n}$. Since $A / A^{2} \cong A^{0} /\left(A^{2}\right)^{0}$ is finite there is an integer $k>0$ such that $k a_{1} \in A^{n}$. But then $k\left(a_{1} a_{2} \cdots a_{n}\right) \in A^{n+1}=0$ which contradicts the fact that $\left(A^{n}\right)^{+}$is infinite cyclic. This means $n=1$ as required.

We can now characterize all semiperfect rings with a cyclic adjoint group. The ring of integers modulo $n$ is denoted by $\boldsymbol{Z}_{n}$.

THEOREM 2. Let $R$ be a semiperfect ring with cyclic adjoint group. Either $R$ is the zero ring on the additive group of integers or $R$ is finite. If $R$ is finite it is a finite direct sum of rings of the following types chosen so that the orders of their adjoint groups are relatively prime in pairs. (See Remark 1 below.)

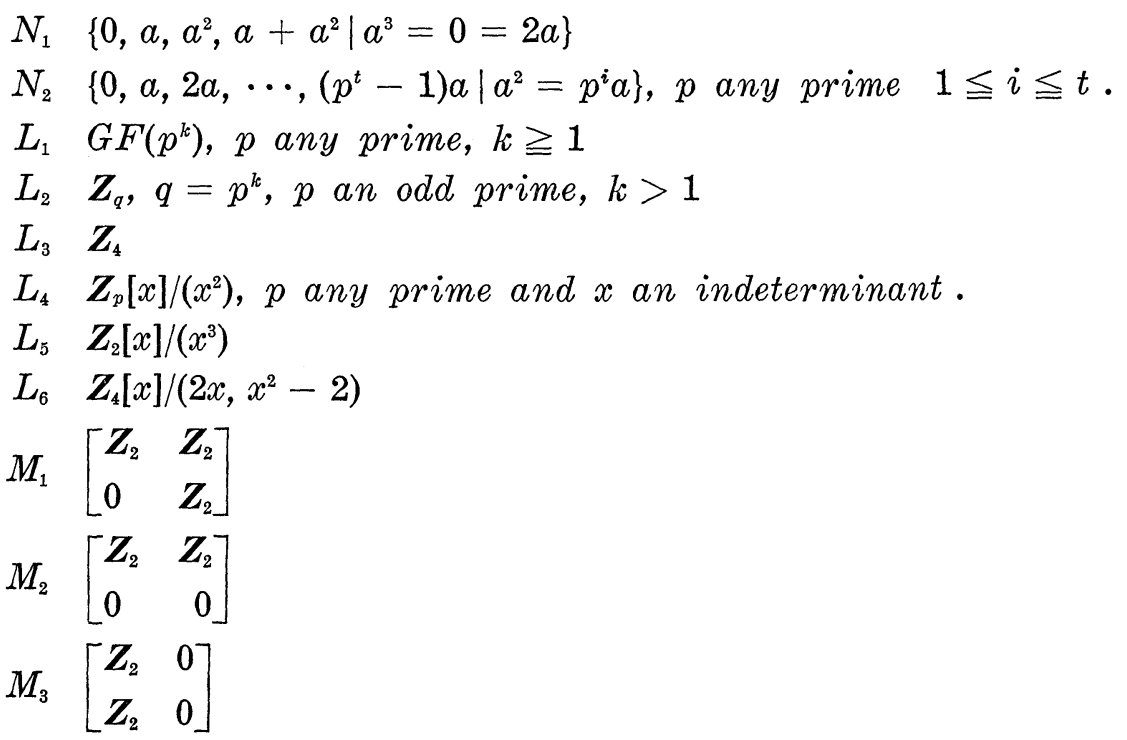

Proof. We note first that Fischer and Eldridge [1] show that every finite ring $A$ with $J(A)=A$ and $A^{0}$ cyclic is a direct sum (see the remark below) of rings of type $N_{1}$ and $N_{2}$. Gilmer [2] shows that each finite local ring with cyclic group of units is of type $L_{i}$ for some $i$.

Now suppose $R$ is semiperfect and $R^{0}$ is cyclic. Consider the decomposition of $R$ given in Theorem 1 . We can apply Lemma 4 to 
the ring $A$. If $A^{+}$is infinite cyclic then $R=A$ is the zero ring on the additive group of integers. If $A$ is finite then $R$ is finite by Corollary 2. In this case the preceding paragraph asserts that the rings $A$ and $T$ in Theorem 2 are finite direct sums of rings of type $N_{i}$ and $L_{j}$. By Theorem 2 of [4] the indecomposable semiperfect ring $S$ is either $\boldsymbol{Z}_{2}$ or the triangular matrix $\operatorname{ring}\left[\begin{array}{ll}\boldsymbol{Z}_{2} & \boldsymbol{Z}_{2} \\ 0 & \boldsymbol{Z}_{2}\end{array}\right]$. In the latter

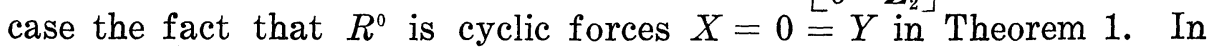
the former case one of $X$ or $Y$ must be zero and the other isomorphic to $\boldsymbol{Z}_{2}^{+}$or zero. Hence the ring $\left[\begin{array}{cc}S & X \\ Y & 0\end{array}\right]$ in Theorem 1 is isomorphic to $Z_{2}$ or one of the rings of type $M_{k}$. This completes the proof.

REMARK 1. As Fischer and Eldridge point out, if $p=2$ the rings of type $N_{2}$ may fail to have a cyclic adjoint group for certain values of $i$. However, this is the only such case.

REMARK 2. The proof that any artinian ring with cyclic adjoint group is finite and has the structure described here is given in [1]. However, the present proof of the way the summands of type $M_{k}$ arise is quite different.

The author would like to thank the referee for suggesting the present proof of Lemma 4.

\section{REFERENCES}

1. I. Fischer and K. E. Eldridge, Artinian rings with a cyclic quasi-regular group, Duke Math. J., 36 (1969), 43-48.

2. R. Gilmer, Finite rings with a cyclic multiplicative group of units, Amer. J. Math., 85 (1963), 447-452.

3. B. J. Mueller, On semiperfect rings, Illinois J. Math., 14 (1970), 464-467.

4. W. K. Nicholson, Semiperfect rings with an Abelian group of units, Pacific J. Math., 49 (1973), 191-198.

5. T. Szele, Nilpotent Artinian rings, Publ. Math. Debrecen, 4 (1955), 71-78.

6. J. F. Watters, On the adjoint group of a radical ring, J. London Math. Soc., 43 (1968), 725-729.

Received May 8, 1973 and in revised form September 25, 1973. This research was supported by N.R.C. (Canada) grant A8075.

University of Calgary, Canada 



\section{PACIFIC JOURNAL OF MATHEMATICS}

\section{EDITORS}

RICHARD ARENS (Managing Editor)

University of California

Los Angeles, California 90024

R. A. Beaumont

University of Washington

Seattle, Washington 98105
J. DugundJI

Department of Mathematics University of Southern California Los Angeles, California 90007

D. Gilbarg AND J. Milgram Stanford University

Stanford, California 94305

\section{ASSOCIATE EDITORS}
E. F. BECKENBACH
B. H. NeUmanN
F. WOLF
K. YOSHIDA

\section{SUPPORTING INSTITUTIONS}

UNIVERSITY OF BRITISH COLUMBIA CALIFORNIA INSTITUTE OF TECHNOLOGY

UNIVERSITY OF CALIFORNIA

MONTANA STATE UNIVERSITY

UNIVERSITY OF NEVADA

NEW MEXICO STATE UNIVERSITY

OREGON STATE UNIVERSITY

UNIVERSITY OF OREGON

OSAKA UNIVERSITY
UNIVERSITY OF SOUTHERN CALIFOF

STANFORD UNIVERSITY

UNIVERSITY OF TOKYO

UNIVERSITY OF UTAH

WASHINGTON STATE UNIVERSITY UNIVERSITY OF WASHINGTON

AMERICAN MATHEMATICAL SOCIET NAVAL WEAPONS CENTER 


\section{Pacific Journal of Mathematics}

\section{Vol. 54, No. 1 \\ May, 1974}

Ralph K Amayo, Engel Lie rings with chain conditions ..................

Bernd Anger and Jörn Lembcke, Hahn-Banach type theorems for hypolinear

functionals on preordered topological vector spaces ..................

Gregory Frank Bachelis and Samuel Ebenstein, On $\Lambda(p)$ sets ................

Harvey Isaac Blau, Indecomposable modules for direct products of finite

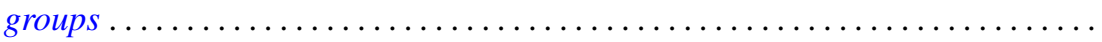

Larry Eugene Bobisud and James Calvert, Singular perturbation of a

time-dependent Cauchy problem in a Hilbert space ................

Walter D. Burgess and Robert Raphael, Abian's order relation and orthogonal

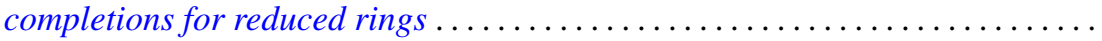

James Diederich, Representation of superharmonic functions mean continuous at

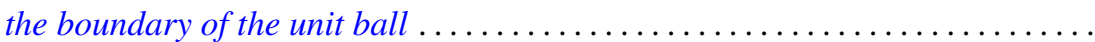

Aad Dijksma and Hendrik S. V. de Snoo, Self-adjoint extensions of symmetric

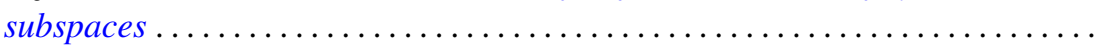

Gustave Adam Efroymson, A Nullstellensatz for Nash rings . . . . . . . . . . . . .

John D. Elwin and Donald R. Short, Branched immersions onto compact orientable surfaces . . . . . . . . . . . . . . . . . . . . . . . . .

John Douglas Faires, Comparison of the states of closed linear

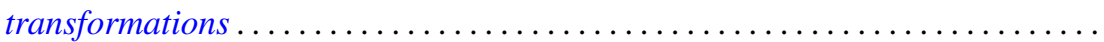

Joe Wayne Fisher and Robert L. Snider, On the von Neumann regularity of rings with regular prime factor rings .

Franklin Takashi Iha, A unified approach to boundary value problems on compact intervals

Palaniappan L. Kannappan and Che Tat $\mathrm{Ng}$, On functional equations connected with directed divergence, inaccuracy and generalized directed divergence

Samir A. Khabbaz and Elias Hanna Toubassi, The module structure of Ext $(F, T)$ over the endomorphism ring of $T$...

Garo K. Kiremidjian, On deformations of complex compact manifolds with boundary.

Dimitri Koutroufiotis, Mappings by parallel normals preserving principal

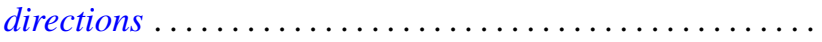

W. K. Nicholson, Semiperfect rings with abelian adjoint group

Norman R. Reilly, Extension of congruences and homomorphisms to translational hulls

Sadahiro Saeki, Symmetric maximal ideals in $M(G)$

Brian Kirkwood Schmidt, On the homotopy invariance of certain functors ...

H. J. Shyr and T. M. Viswanathan, On the radicals of lattice-ordered rings ...

Indranand Sinha, Certain representations of infinite group algebras ...

David Smallen, The group of self-equivalences of certain complexes ...

Kalathoor Varadarajan, On a certain problem of realization in homotopy

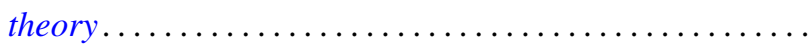

